\title{
Fifteen year survival of patients presenting with hypertension to a hospital clinic
}

\author{
Christopher J. Bulpitt, Pauline F. Bulpitt, Margot Daymond, Kirsten Hartley and \\ Colin T. Dollery
}

The Department of Clinical Pharmacology, Royal Postgraduate Medical School, Ducane Road, London W12, UK.

\begin{abstract}
Summary: The survival has been determined for the 404 patients who presented to the Hammersmith Hospital Hypertension Clinic during the years 1962 to 1966 and in whom the untreated blood pressure was known. The fifteen year survival ranged from $72 \%$ for young men aged $30-49$ at presentation to $27 \%$ for men aged 60-69. Sixty-eight percent of the deaths were cardiovascular or renal, $33 \%$ of all deaths were from ischaemic heart disease (IHD), $17 \%$ from stroke and $3 \%$ from renal causes. Death from any cause was predicted with statistical significance by age, the presence of accelerated or malignant hypertension, impaired renal function, smoking at presentation and systolic blood pressure. Death was not predicted by hypokalaemia, hyperuricaemia (after adjusting for renal function) and obesity.
\end{abstract}

\section{Introduction}

The drug treatment of moderate or severe hypertension is usually for life and it is important to determine the long term survival of such patients. Survival was determined for the patients who presented to the Hammersmith Hospital Hypertension Clinic between the years 1962 to 1966 . These years were selected as marking the beginning of an era of more modern antihypertensive treatment, the use of ganglion blocking drugs having been discontinued and the beta-adrenoceptor blocking drugs having been introduced towards the end of that 5 year period. This report describes the characteristics of this cohort of patients and their survival according to age, sex, untreated and treated blood pressure, smoking habits, renal function, plasma potassium, serum cholesterol and uric acid.

\section{Methods}

The records of the Hammersmith Hospital Hypertension Clinic include the names of 404 patients who first attended the clinic for treatment in the years 1962 to 1966 and who had an untreated diastolic blood pressure known to be equal to or greater than $90 \mathrm{~mm} \mathrm{Hg}$. The patients were registered with the Office of Population Censuses and Surveys (OPCS) in

Correspondence: C.J. Bulpitt, M.Sc., M.D., F.R.C.P., Department of Epidemiology, London School of Hygiene and Tropical Medicine, Keppel Street, London WC1E 7HT Accepted: 12 August 1985 order to receive a copy of any death certificate prior to July 1981 . Outcome could not be determined in 34 patients. Information concerning visits up to July 1977 was extracted from the clinic notes and included the following data: age, sex, race, untreated blood pressure, smoking habits, family history of hypertension, retinal appearance, weight and biochemical results at presentation. This information was not available for all patients and the completeness of data is indicated in the tables. Information on follow-up was also retrieved and included blood pressure measurements during treatment, measurements of plasma urea and potassium, serum cholesterol and uric acid. The methods of study have been described previously and certain results for 205 patients who received methyldopa have been reported (Dollery et al., 1984).

\section{Statistical methods}

The survival of the patients was analysed using standard life table techniques (Berkson \& Gage, 1950). When different groups were compared for survival the nonparametric test of Lee \& Desu (1972) was employed. When different groups were compared for characteristics other than survival, unpaired $t$ tests, analysis of variance and chi-squared techniques were employed as appropriate. In order to determine the independent effects of the different variables on survival, the fact of death was employed as the $Y$ variable in multiple regression equations.

Life tables were also calculated separately for a death from ischaemic heart disease (IHD), stroke and 
renal disease and cancer. The end point in each instance was a mention of the cause of death on the death certificate. Patients who died from another cause of death, other than the cause mentioned, were censored (assumed lost to follow-up) at the date of death.

\section{Results}

The characteristics of the patients at presentation

Table I gives the characteristics of the patients at presentation. Their average age was 50 years with a range from 13 to 84 . By 1985 standards they were a severely hypertensive group, average untreated lying pressure being $210 \mathrm{~mm} \mathrm{Hg}$ systolic and $122 \mathrm{~mm} \mathrm{Hg}$ diastolic. Over $4 \%$ had malignant hypertension and $21 \%$ accelerated hypertension (cotton wool spots or haemorrhages in the retina without papilloedema). Sixty-three percent of the men and $31 \%$ of the women smoked.

\section{Characteristics of the patients during follow-up}

Table I also gives the characteristics of the patients during follow-up including the average treated blood pressure for all patients alive and attending the clinic at the time of the second and fifth year of follow-up and the average biochemical results during follow-up. Blood pressure control tended to be better in men than in women.

\section{Causes of death}

The underlying cause of death for the 158 patients who died was determined from the death certificates. Sixtyeight percent of the deaths were cardiovascular or renal. Smoking related diseases such as lung cancer tended to be more common in males. Thirty two percent of deaths were from ischaemic heart disease in men and $34 \%$ in women (ICD 8th revision codes $410-414), 13 \%$ of deaths were from stroke in men and $23 \%$ in women (ICD $430-438$ ), $3 \%$ from renal causes in both men and women (ICD 580-599) and $16 \%$ of

Table I Characteristics of patients at presentation and information extracted from the records during follow-up

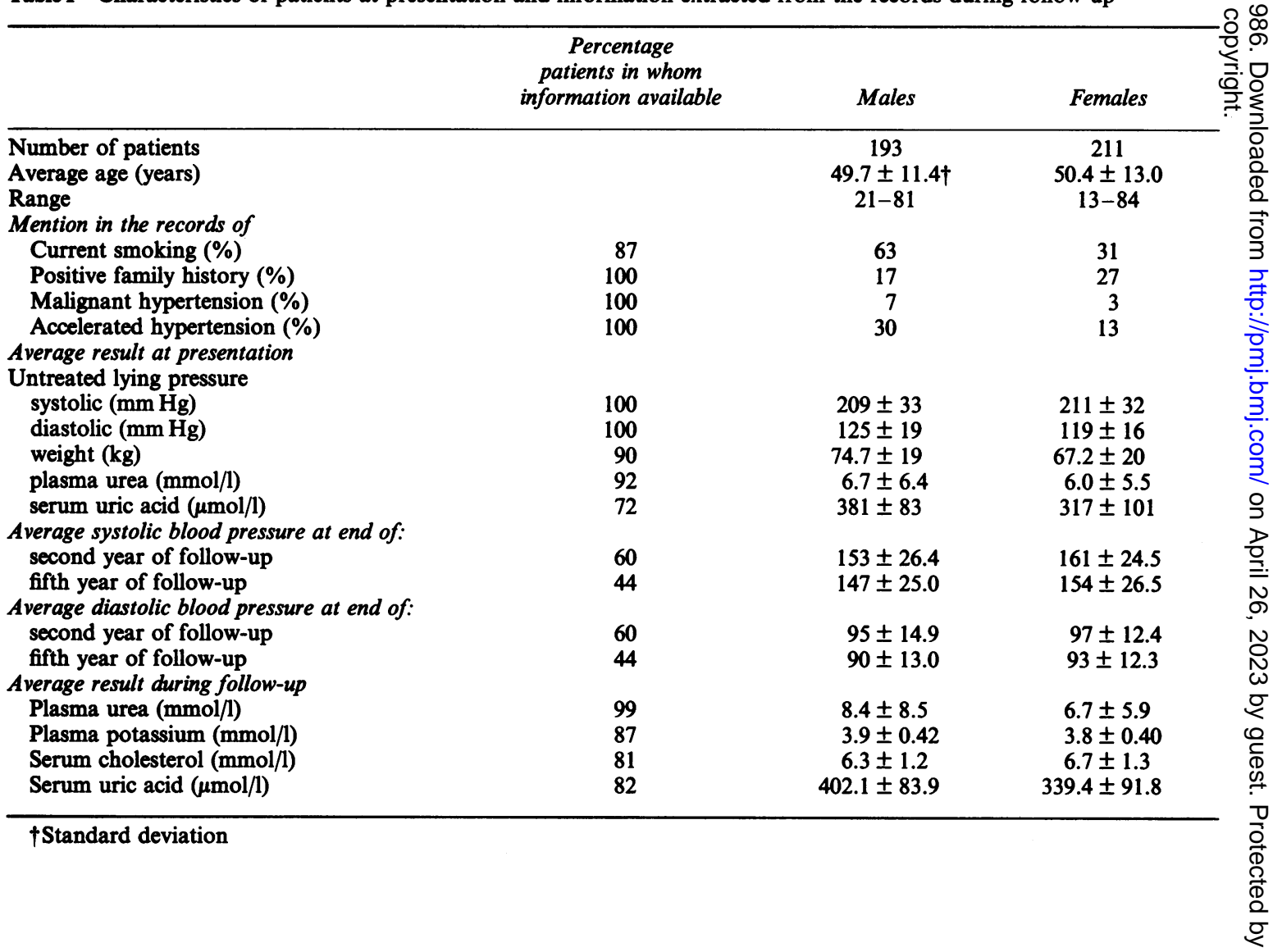




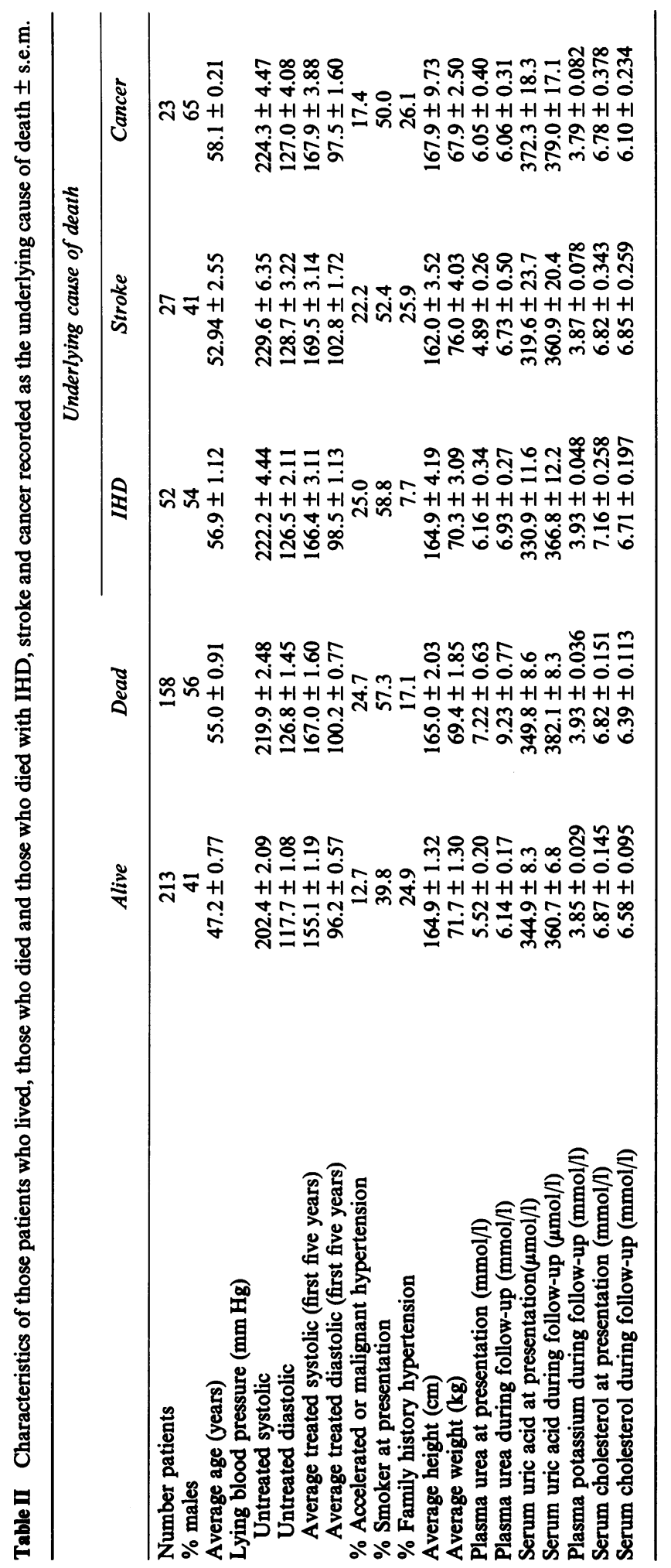


men and $13 \%$ of women died from other cardiovascular causes (remainder of 390-459). Cancer deaths excluding lung cancer accounted for $10 \%$ of the deaths both men and women. Lung cancer deaths accounted for $7 \%$ and $1 \%$ respectively.

\section{Determinants of survival}

Table II gives certain results according to whether the patients lived or died. The results are not adjusted for age and other variables but they are so adjusted in Section 7 below. Table II is available for both sexes separately on application to the authors.

1. Blood pressure, presence of accelerated or malignant hypertension

Patients who died had higher average untreated systolic and diastolic pressures than those who survived, higher average treated systolic and diastolic pressures and were more likely to have had accelerated or malignant hypertension $(P<0.01$ for all comparisons). These associations were true after adjusting for age (Section 7). Patients dying from a stroke had the highest untreated and treated levels of systolic and diastolic pressure.

2. Renal function at presentation

Patients who died had a higher average plasma urea at presentation $(P<0.01)$ and during follow-up $(P<0.001)$. This was true even when deaths from renal causes were excluded and after adjusting for age.

3. Serum uric acid

The average serum uric acid tended to be higher during follow-up in patients who died. This association was not present after adjusting for plasma urea.

\section{Plasma potassium}

The average plasma potassium tended to be higher in those who died but this appeared to be due to deterioration in renal function in a proportion of these patients. After adjusting for renal function or excluding those with renal failure neither a low nor a high serum potassium was associated with death. The table of average plasma potassium according to outcome and 4 categories of plasma urea is available from the authors.

5. Serum cholesterol and weight

For total mortality, the serum cholesterol either at presentation or during follow-up, was not an important predictor of death. However, serum cholesterol during follow-up was higher in men who died from cardiovascular diseases than those who died from cancer $(P<0.01)$. Neither over- nor under-weight were important predictors of survival.

6. Smoker at presentation

Smoking cigarettes at presentation was more common among those who died than those who lived
$(P<0.01)$. This was also statistically significant when deaths from ischaemic heart disease were considered on their own $(P<0.05)$.

7. Prediction of death after adjusting for age and other variables

After adjusting for age and sex the important predictors were, ranked in order of the partial correlation coefficients, average urea during follow-up $(r=0.25, P<0.001)$, untreated diastolic pressure $(r=0.22, P<0.001)$, five year average treated systolic pressure $(r=0.20, P<0.001)$, un treated systolic pressure $(r=0.19, P<0.001)$, presenting plasma urea $(r=0.18, P<0.01)$, five year average treated diastolic pressure $(r=0.16$, $P<0.01$ ), presence of accelerated or malignant hypertension $(r=0.15, P<0.01)$, and smoking at presentation $(r=0.13, P=0.01)$. Partial correlations within the range +0.05 and -0.05 were observed for uric acid and cholesterol at presentation and a family history of hypertension. Death was predicted on the basis of all the following variables in a multiple regression equation $(\beta=$ partial regression coefficient): age $(\beta=0.014$, $P<0.001)$, smoking $(\beta=0.14, P<0.01)$, average urea during follow up $(\beta=0.013, P<0.01)$ and untreated diastolic pressure $(\beta=0.0051$, $P<0.001$ ), constant $=-0.217$. Being male or having malignant or accelerated hypertension did not make any important additional contribu-0 tion to this equation $(t=0.8$ and 0.1 to enter respectively).

8. Time of death from renal failure, stroke, IHD and cancer

When considering deaths in the first year, 4 of the 5 renal deaths occurred during this period. The probability of a renal death in the first year was 0.0009 when computed from the life table analysis. Eleven stroke deaths occurred during the first 5 years of follow-up (average probability $0.0006 /$ year) and 15 in the next 10 years (average probability $0.0005 /$ year). Deaths from IHD were also constant over time. Ten IHD deaths occurred in men in the first 5 years (average probability $0.0011 /$ year) and 17 in the next 10 years (average probability $0.0012 /$ year). Eighteen IHD deaths occurred in women in the first 5 years (average probability $0.0007 /$ year) and 14 in the next ten years (average probability also $0.0007 /$ year).

9. Length of survival and the effect of treatment Table III reports the 15 year survival of patients from presentation in three age groups. For comparative purposes the survival for the general population in England and Wales is reported for the same period of time. The 15 year survival of all patients ranged from $72 \%$ for men aged $30-49$ at presentation to $27 \%$ for men aged 60-69. The younger patients, both men and women, had the 
Table III Five, ten and fifteen year survival from presentation (\%) in male and female patients in 3 age groups and the corresponding figures for the general population of England and Wales (cohort analysis for age 42, 53 and 63 at start)

\begin{tabular}{|c|c|c|c|c|}
\hline & \multicolumn{2}{|c|}{ Men } & \multicolumn{2}{|c|}{ Women } \\
\hline & (1) & $\begin{array}{c}\text { General } \\
\text { population (2) }\end{array}$ & (1) & $\begin{array}{c}\text { General } \\
\text { population (2) }\end{array}$ \\
\hline \multicolumn{5}{|l|}{ Age $30-49$} \\
\hline$n$ & 79 & & 80 & \\
\hline 5 year survival & 85 & 98 & 85 & 99 \\
\hline 10 year survival & 77 & 97 & 82 & 98 \\
\hline 15 year survival & 72 & 92 & 70 & 95 \\
\hline SE (15 year) & 5.5 & & 5.5 & \\
\hline Relative risk ( 15 year) & 3.5 & & 6.0 & \\
\hline Attributable risk (/100/15 years) & 20 & & 25 & \\
\hline \multicolumn{5}{|l|}{ Age 50-59 } \\
\hline$n$ & 66 & & 74 & \\
\hline 5 year survival & 84 & 93 & 96 & 97 \\
\hline 10 year survival & 68 & 80 & 85 & 91 \\
\hline 15 year survival & 50 & 65 & 75 & 81 \\
\hline SE (15 years) & 6.4 & & 5.1 & \\
\hline Relative risk ( 15 years) & 1.4 & & 1.3 & \\
\hline Attributable risk (/100/15 years) & 15 & & 6 & \\
\hline \multicolumn{5}{|l|}{ Age 60-69 } \\
\hline$n$ & 35 & & 30 & \\
\hline 5 year survival & 66 & 80 & 66 & 90 \\
\hline 10 year survival & 46 & 57 & 55 & 75 \\
\hline 15 year survival & 27 & 33 & 47 & 54 \\
\hline SE (15 years) & 7.7 & & 9.4 & \\
\hline Relative risk ( 15 years) & 1.1 & & 1.2 & \\
\hline Attributable risk $(/ 100 / 15$ years $)$ & 6 & & 7 & \\
\hline
\end{tabular}

Relative risk at 15 years $\frac{100-(1)}{100-(2)}$

Risk attributable to hypertension $=(2)-(1) / 100 / 15$ years

SE $(15$ years $)=$ standard error of cumulative \% surviving at 15 years.

highest relative and attributable risks from presenting with hypertension.

\section{Discussion}

The treatment of hypertension in these patients presenting in the 1960s did not confer the same expectation of life as in the general population. During the fifteen years the relative risk of dying for both male and female patients under 60 years of age ranged from 1.3 to 6.0. It may be argued that the relative risk is a poor indicator as the general population of England and Wales may not be an appropriate comparison group for patients referred to the Hammersmith Hypertension Clinic, and moreover, the relative risk must approach unity in the elderly when many die over 15 years. However, both relative risk and the risk attributable to being a patient was markedly increased in men and women under the age of 50, compared with the mortality rate in the general population. An excess of between $20 \%$ and $25 \%$ of those under the age of 50 died over the 15 years of observation.

In the 1950 s mortality was even greater as $54 \%$ of patients attending the Hammersmith Clinic presented with accelerated or malignant hypertension and $44 \%$ died from uraemia (Breckenridge et al., 1970). In the present study from the $1960 \mathrm{~s}, 37 \%$ of men and $16 \%$ of women had malignant or accelerated hypertension at presentation, but only $3 \%$ of males and females died of renal disease during 15 years of follow-up. The decline in uraemic deaths must be partly due to dialysis and transplantation, but the large decrease in the proportion presenting with accelerated and malignant hypertension suggests that these conditions were becoming rarer in the general population, either due to a change in the severity or nature of the underlying disease, or to early and preventive treatment.

Despite fears that diuretic treatment may increase IHD rates (Multiple Risk Factor Intervention Trial Research Group, 1982), the death rate for IHD was not observed to increase with time and was fairly 
stable year by year. The present study does not suggest any worsening in the survival of hypertensive patients during long-term follow-up and the recent reports from the European Working Party on High Blood Pressure in the Elderly (Amery et al., 1985) and Medical Research Council Working Party (1985) trials do not suggest an excessive IHD risk from diuretic treatment. The patients in the present study were far more severely hypertensive than in either of these trials. Both trials showed a $40 \%$ or greater reduction in stroke events, but owing to the high incidence of stroke in the elderly, only 90 patients had to be treated for one year to save one stroke event in the EWPHE trial (incidence in placebo group $2.9 \%$ /year) against 850 for one year in the MRC trial (placebo incidence $0.3 \% /$ year). The corresponding number of patients to prevent one death from stroke were 170 and 5,000 patients treated for one year in the EWPHE and MRC trials respectively.

The average age of the patients in the present study was comparable with that in the MRC trial. However, the stroke death rate on active treatment in our study was much higher, suggesting that the number of stroke deaths prevented by every year of treatment may be greater than in the MRC trial.

The data support the present strategy of rigorous anti-smoking advice, but provide less cause for concern with respect to other potential risk factors such as obesity, hypokalaemia and hyperuricaemia. Patients with hypokalaemia appeared to survive as long as those who did not have this condition. Hyperuricaemia also did not appear to be a predictor of death once renal function had been taken into account. The patients who died were not more obese than the survivors.

Serum cholesterol was higher in those men dying from cardiovascular causes than those dying of cancer. Overall total mortality was not related to serum cholesterol, a finding reported in patients presenting in the 1970s (Bulpitt et al., 1979).

The severely hypertensive group of patients who began treatment in the early 1960 s achieved a remarkably long survival but they still suffered a substantial excess of cardiovascular mortality. The patients who died had more severe hypertension, more cigarette smokers, a greater degree of renal damage, were older and more likely to be of male sex. Early treatment of moderately severe hypertension should reduce the degree of vascular damage that occurs before the treatment is begun but there is still much to be done if a goal of normal life expectancy is to be attained.

\section{Acknowledgements}

We are grateful to Merck Sharp and Dohme International for a grant to make the study possible.

\section{References}

AMERY, A., BIRKENHAGER, W., BRIXKO, P., BULPITT, C.J. and members of the Working Party. (1985). Mortality and morbidity results from the European Working Party on high blood pressure in the elderly trial. Lancet, i, 1349.

BERKSON, J. \& GAGE, R. (1950). Calculation of survival rates for cancer. Proceedings of Mayo Clinic, 25, 270.

BRECKENRIDGE, A., DOLLERY, C.T. \& PARRY, E.H.O. (1970). Prognosis of treated hypertension. Quarterly Journal of Medicine, 39, 411.

BULPITT, C.J., BEILIN, L.J., CLIFTON, P., COLES, E.C., DOLLERY, C.T., GEAR, J.S., HARPER, G.S., JOHNSON, B.F. \& MUNRO-FAURE, A.D. (1979). Risk factors for death in treated hypertensive patients. Report from the DHSS Hypertension Care Computing Project. Lancet, ii, 134.

DOLLERY, C.T., HARTLEY, K., BULPITT, P.F., DAYMOND M. \& BULPITT, C.J. (1984). Fifteen year survival of patients beginning treatment with methyldopa between 1962 and 1966. Hypertension, 6, II, 82.

LEE, E. \& DESU, M. (1972). A computer program for comparing $\mathrm{K}$ samples with right-censored data. Computer Programs in Biomedicine, 2, 315.

MEDICAL RESEARCH COUNCIL WORKING PARTY (1985). MRC trial of treatment of mild hypertension: principal results. British Medical Journal, $291,97$.

MULTIPLE RISK FACTOR INTERVENTION TRIAL RESEARCH GROUP (1982). Multiple risk factor intervention trial. Risk factor changes and mortality results. Journal of the American Medical Association, 248, 1465. 